PROCEEDINGS OF THE

AMERICAN MATHEMATICAL SOCIETY

Volume 131, Number 7, Pages 1989-1998

S 0002-9939(03)06941-7

Article electronically published on February 11, 2003

\title{
ON THE IRRATIONALITY OF A CERTAIN MULTIVARIATE $q$ SERIES
}

\author{
PETER B. BORWEIN AND PING ZHOU
}

(Communicated by David E. Rohrlich)

\begin{abstract}
We prove that for integers $q>1, m \geq 1$ and positive rationals $r_{1}, r_{2}, \cdots, r_{m} \neq q^{j}, j=1,2, \cdots$, the series

$$
\sum_{j=1}^{\infty} \frac{q^{-j}}{\left(1-q^{-j} r_{1}\right)\left(1-q^{-j} r_{2}\right) \cdots\left(1-q^{-j} r_{m}\right)}
$$
\end{abstract}

is irrational. Furthermore, if all the positive rationals $r_{1}, r_{2}, \cdots, r_{m}$ are less than $q$, then the series

is also irrational.

$$
\sum_{j_{1}, \cdots, j_{m}=0}^{\infty} \frac{r_{1}^{j_{1}} \cdots r_{m}^{j_{m}}}{q^{j_{1}+\cdots+j_{m}+1}-1}
$$

\section{IntroduCtion AND RESUlts}

The main result of this paper is the following theorem:

Theorem 1.1. If $q$ is an integer greater than one, $m$ is a positive integer, and $r_{1}, r_{2}, \cdots, r_{m}$ are any positive rationals such that $r_{1}, r_{2}, \cdots, r_{m} \neq q^{j}, j=1,2, \cdots$, then the series

$$
\sum_{j=1}^{\infty} \frac{q^{-j}}{\left(1-q^{-j} r_{1}\right)\left(1-q^{-j} r_{2}\right) \cdots\left(1-q^{-j} r_{m}\right)}
$$

is irrational. Furthermore, if all the positive rationals $r_{1}, r_{2}, \cdots, r_{m}$ are less than $q$, then the series

$$
\sum_{j_{1}, \cdots, j_{m}=0}^{\infty} \frac{r_{1}^{j_{1}} \cdots r_{m}^{j_{m}}}{q^{j_{1}+\cdots+j_{m}+1}-1}
$$

is also irrational.

This generalizes the irrationality results of the single variable case proved in Borwein [3], Erdös [6], and Erdös and Graham [7]. The approach is via Padé approximants. These provide, when appropriately specialized, rational approximations that are "too good" to allow for rationality. These methods are also used in Borwein and Zhou [4], Mahler [9], Chudnovsky and Chudnovsky [5], Wallisser [10], and Zhou and Lubinsky [11. Unfortunately the methods are not sufficiently general

Received by the editors December 18, 2001.

2000 Mathematics Subject Classification. Primary 11J72.

The second author's research was supported in part by NSERC of Canada. 
to allow a unified treatment and each new class of functions requires considerable additional work.

As in [4] we use the standard $q$ analogues of factorials and binomial coefficients. The $q$-factorial is

$$
[n]_{q} !:=[n] !:=\frac{\left(1-q^{n}\right)\left(1-q^{n-1}\right) \cdots(1-q)}{(1-q)^{n}},
$$

where $[0]_{q} !:=1$. The $q$-binomial coefficient is

$$
\left[\begin{array}{l}
n \\
k
\end{array}\right]_{q}:=\left[\begin{array}{l}
n \\
k
\end{array}\right]:=\frac{[n] !}{[k] ! \cdot[n-k] !} .
$$

As

$$
q^{i}-1=(q-1)\left(q^{i-1}+q^{i-2}+\cdots+1\right), \quad i \geq 1,
$$

we have

$$
\lim _{q \rightarrow 1}[n]_{q} !=n ! \quad \text { and } \quad \lim _{q \rightarrow 1}\left[\begin{array}{l}
n \\
k
\end{array}\right]_{q}=\left(\begin{array}{l}
n \\
k
\end{array}\right) .
$$

Note that (see Borwein [3])

$$
\begin{gathered}
{[n]_{q^{-1}} !=q^{-n(n-1) / 2}[n] !,} \\
{\left[\begin{array}{l}
n \\
k
\end{array}\right]_{-q}=q^{-k(n-k)}\left[\begin{array}{l}
n \\
k
\end{array}\right],} \\
\prod_{\substack{h=0 \\
h \neq k}}^{n}\left(q^{-k}-q^{-h}\right)=(-1)^{n-k} q^{-k(k-1) / 2-n(n+1) / 2}[n-k] ![k] !(1-q)^{n},
\end{gathered}
$$

and (see Gasper and Rahman [8]) for $|t|<q^{-n}$,

$$
\frac{1}{\prod_{k=0}^{n}\left(t-q^{-k}\right)}=(-1)^{n+1} q^{n(n+1) / 2} \sum_{l=0}^{\infty}\left[\begin{array}{c}
n+l \\
l
\end{array}\right] t^{l} .
$$

We prove some properties of approximants to a related function in section 2, and use those properties to prove Theorem 1.1 in section 3 .

\section{Some Results on A RELATEd FUnCtion}

Let $q>1,\left|x_{1}\right|, \cdots,\left|x_{m}\right|<q$, and integer $m \geq 1$, and let

$$
L^{*}\left(x_{1}, \cdots, x_{m}\right):=\sum_{j_{1}, \cdots, j_{m}=0}^{\infty} \frac{x_{1}^{j_{1}} \cdots x_{m}^{j_{m}}}{q^{j_{1}+\cdots+j_{m}+1}-1} .
$$

For $m=1$, and $|x|<1$,

$$
\begin{aligned}
\lim _{q \rightarrow 1}(q-1) L^{*}(x) & =\lim _{q \rightarrow 1} \sum_{j=0}^{\infty} \frac{(q-1) x^{j}}{q^{j+1}-1} \\
& =\sum_{j=0}^{\infty} \frac{x^{j}}{j+1} \\
& =\frac{1}{x} \ln (1-x) .
\end{aligned}
$$


So we call $L^{*}\left(x_{1}, \cdots, x_{m}\right)$ a multivariate $q$ analogue of log. Now for $k \geq 1$ integer and $\left|x_{1}\right|, \cdots,\left|x_{m}\right|<q$, as

$$
\begin{aligned}
L^{*}\left(q^{-1} x_{1}, \cdots, q^{-1} x_{m}\right) & =\sum_{j_{1}, \cdots, j_{m}=0}^{\infty} \frac{q^{-\left(j_{1}+\cdots+j_{m}\right)} x_{1}^{j_{1}} \cdots x_{m}^{j_{m}}}{q^{j_{1}+\cdots+j_{m}+1}-1} \\
& =\sum_{j_{1}, \cdots, j_{m}=0}^{\infty} \frac{\left(1-q^{j_{1}+\cdots+j_{m}+1}+q^{j_{1}+\cdots+j_{m}+1}\right) x_{1}^{j_{1}} \cdots x_{m}^{j_{m}}}{q^{j_{1}+\cdots+j_{m}}\left(q^{j_{1}+\cdots+j_{m}+1}-1\right)} \\
& =\sum_{j_{1}, \cdots, j_{m}=0}^{\infty} \frac{q x_{1}^{j_{1}} \cdots x_{m}^{j_{m}}}{q^{j_{1}+\cdots+j_{m}+1}-1}-\sum_{j_{1}, \cdots, j_{m}=0}^{\infty} \frac{x_{1}^{j_{1}} \cdots x_{m}^{j_{m}}}{q^{j_{1}+\cdots+j_{m}}} \\
& =q L^{*}\left(x_{1}, \cdots, x_{m}\right)-\frac{1}{\left(1-q^{-1} x_{1}\right) \cdots\left(1-q^{-1} x_{m}\right)},
\end{aligned}
$$

we have

$$
\begin{aligned}
L^{*}\left(q^{-k} x_{1}, \cdots, q^{-k} x_{m}\right) & =q^{k} L^{*}\left(x_{1}, \cdots, x_{m}\right)-\sum_{j=1}^{k} \frac{q^{k-j}}{\left(1-q^{-j} x_{1}\right) \cdots\left(1-q^{-j} x_{m}\right)} \\
(2.2) & =: q^{k} L^{*}\left(x_{1}, \cdots, x_{m}\right)-S_{k}\left(x_{1}, \cdots, x_{m}\right),
\end{aligned}
$$

where

$$
S_{k}\left(x_{1}, \cdots, x_{m}\right):=\sum_{j=1}^{k} \frac{q^{k-j}}{\left(1-q^{-j} x_{1}\right) \cdots\left(1-q^{-j} x_{m}\right)} .
$$

From (2.2), we have

$$
L^{*}\left(x_{1}, \cdots, x_{m}\right)=q^{-k} L^{*}\left(q^{-k} x_{1}, \cdots, q^{-k} x_{m}\right)+\sum_{j=1}^{k} \frac{q^{-j}}{\left(1-q^{-j} x_{1}\right) \cdots\left(1-q^{-j} x_{m}\right)},
$$

and then

$$
\begin{aligned}
L^{*}\left(x_{1}, \cdots, x_{m}\right)= & \lim _{k \rightarrow \infty} q^{-k} L^{*}\left(q^{-k} x_{1}, \cdots, q^{-k} x_{m}\right) \\
& +\lim _{k \rightarrow \infty} \sum_{j=1}^{k} \frac{q^{-j}}{\left(1-q^{-j} x_{1}\right) \cdots\left(1-q^{-j} x_{m}\right)} \\
= & \sum_{j=1}^{\infty} \frac{q^{-j}}{\left(1-q^{-j} x_{1}\right) \cdots\left(1-q^{-j} x_{m}\right)} .
\end{aligned}
$$

Now let $q>1, x_{1}, \cdots, x_{m} \neq q^{j}, j=1,2, \cdots$, and integer $m \geq 1$, and let

$$
L\left(x_{1}, \cdots, x_{m}\right):=\sum_{j=1}^{\infty} \frac{q^{-j}}{\left(1-q^{-j} x_{1}\right) \cdots\left(1-q^{-j} x_{m}\right)} .
$$

Then $L\left(x_{1}, \cdots, x_{m}\right)$ is an extension of $L^{*}\left(x_{1}, \cdots, x_{m}\right)$, i.e.

$$
L\left(x_{1}, \cdots, x_{m}\right)=L^{*}\left(x_{1}, \cdots, x_{m}\right), \text { for }\left|x_{1}\right|, \cdots,\left|x_{m}\right|<q .
$$

It is easy to see that we also have the following functional equation for $L\left(x_{1}, \cdots, x_{m}\right)$ :

$$
L\left(q^{-k} x_{1}, \cdots, q^{-k} x_{m}\right)=q^{k} L\left(x_{1}, \cdots, x_{m}\right)-S_{k}\left(x_{1}, \cdots, x_{m}\right),
$$

where $k \geq 1$ is an integer and $S_{k}\left(x_{1}, \cdots, x_{m}\right)$ is defined by (2.3). Now we prove some properties of the function $L\left(x_{1}, \cdots, x_{m}\right)$. 
Theorem 2.1. Let $n \geq 0$ be an integer, and let $L\left(x_{1}, \cdots, x_{m}\right), S_{k}\left(x_{1}, \cdots, x_{m}\right)$ be defined by (2.5) and (2.3) respectively. Let

$$
R_{n}\left(x_{1}, \cdots, x_{m}\right):=\prod_{j=1}^{n}\left(\left(1-q^{-j} x_{1}\right) \cdots\left(1-q^{-j} x_{m}\right)\right)
$$

and

$$
I\left(x_{1}, \cdots, x_{m}\right):=\frac{R_{n}\left(x_{1}, \cdots, x_{m}\right)}{2 \pi i} \int_{\Gamma} \frac{L\left(t x_{1}, \cdots, t x_{m}\right) d t}{\left(\prod_{k=0}^{n}\left(t-q^{-k}\right)\right) t^{n+1}},
$$

where $\Gamma$ is a circular contour containing $0, q^{-n}, \cdots, q^{0}$, and let

$$
\begin{aligned}
& Q\left(x_{1}, \cdots, x_{m}\right):=\frac{q^{n(n+1) / 2}}{(1-q)^{n}[n] !} \sum_{k=0}^{n}(-1)^{n-k}\left[\begin{array}{l}
n \\
k
\end{array}\right] q^{n k+k(k+1) / 2} R_{n}\left(x_{1}, \cdots, x_{m}\right), \\
& P\left(x_{1}, \cdots, x_{m}\right):=\frac{q^{n(n+1) / 2}}{(1-q)^{n}[n] !} \sum_{k=0}^{n}(-1)^{n-k}\left[\begin{array}{l}
n \\
k
\end{array}\right] q^{n k+k(k+1) / 2} \\
& \cdot R_{n}\left(x_{1}, \cdots, x_{m}\right) S_{k}\left(x_{1}, \cdots, x_{m}\right) \\
& +\frac{R_{n}\left(x_{1}, \cdots, x_{m}\right)}{n !} \frac{d^{n}}{d t^{n}}\left\{\frac{L\left(t x_{1}, \cdots, t x_{m}\right)}{\prod_{k=0}^{n}\left(t-q^{k}\right)}\right\}_{t=0} .
\end{aligned}
$$

Then

(i)

$$
I\left(x_{1}, \cdots, x_{m}\right)=Q\left(x_{1}, \cdots, x_{m}\right) L\left(x_{1}, \cdots, x_{m}\right)+P\left(x_{1}, \cdots, x_{m}\right)
$$

(ii)

$$
q^{(m-1) n(n+1) / 2}\left(\prod_{j=1}^{n}\left(q^{j}-1\right)\right) Q\left(x_{1}, \cdots, x_{m}\right) \in \mathbb{Z}\left[q, x_{1}, \cdots, x_{m}\right],
$$

where $\mathbb{Z}\left[q, x_{1}, \cdots, x_{m}\right]$ is the set of polynomials in $q, x_{1}, \cdots, x_{m}$ with integer coefficients;

(iii)

$$
q^{(m-1) n(n+1) / 2}\left(\prod_{j=1}^{n+1}\left(q^{j}-1\right)\right) P\left(x_{1}, \cdots, x_{m}\right) \in \mathbb{Z}\left[q, x_{1}, \cdots, x_{m}\right]
$$

(iv) for $n \in \mathbb{N}$ fixed, and $0<\left|x_{1}\right|, \cdots,\left|x_{m}\right|<q$,

$$
\left|I\left(x_{1}, \cdots, x_{m}\right)\right| \leq \frac{c_{q}}{q^{2 m n(n+1)}},
$$

where $c_{q}$ is a constant depending only on $q, m$, and $x_{1}, \cdots, x_{m}$. 
Proof of Theorem 2.1. Proof of (i). We can see that the integrand in (2.9) has simple poles at $t=q^{0}, q^{-1}, \cdots, q^{-n}$, and a pole of order $n+1$ at $t=0$, inside the contour $\Gamma$. By the residue theorem and the functional equation (2.7), and (1.6), we have

$$
\begin{aligned}
& I\left(x_{1}, \cdots, x_{m}\right)=\frac{R_{n}\left(x_{1}, \cdots, x_{m}\right)}{2 \pi i} \int_{\Gamma} \frac{L\left(t x_{1}, \cdots, t x_{m}\right) d t}{\left(\prod_{k=0}^{n}\left(t-q^{-k}\right)\right) t^{n+1}} \\
&=R_{n}\left(x_{1}, \cdots, x_{m}\right) \sum_{k=0}^{n} \frac{L\left(q^{-k} x_{1}, \cdots, q^{-k} x_{m}\right)}{\left(\prod_{\substack{h=0 \\
h \neq k}}^{n}\left(q^{-k}-q^{-h}\right)\right) q^{-k(n+1)}} \\
&+\frac{R_{n}\left(x_{1}, \cdots, x_{m}\right)}{n !} \frac{d^{n}}{d t^{n}}\left\{\frac{L\left(t x_{1}, \cdots, t x_{m}\right)}{\prod_{k=0}^{n}\left(t-q^{-k}\right)}\right\}_{t=0} \\
&= \frac{q^{n(n+1) / 2} R_{n}\left(x_{1}, \cdots, x_{m}\right)}{(1-q)^{n}[n] !} \sum_{k=0}^{n}(-1)^{n-k}\left[\begin{array}{l}
n \\
k
\end{array}\right] q^{n k+k(k+1) / 2} q^{k} L\left(x_{1}, \cdots, x_{m}\right) \\
&-\frac{q^{n(n+1) / 2} R_{n}\left(x_{1}, \cdots, x_{m}\right)}{(1-q)^{n}[n] !} \sum_{k=0}^{n}(-1)^{n-k}\left[\begin{array}{l}
n \\
k
\end{array}\right] q^{n k+k(k+1) / 2} S_{k}\left(x_{1}, \cdots, x_{m}\right) \\
&+\frac{R_{n}\left(x_{1}, \cdots, x_{m}\right)}{n !} \frac{d^{n}}{d t^{n}}\left\{\frac{L\left(t x_{1}, \cdots, t x_{m}\right)}{\prod_{k=0}^{n}\left(t-q^{-k}\right)}\right\}_{t=0} \\
&= Q\left(x_{1}, \cdots, x_{m}\right) L\left(x_{1}, \cdots, x_{m}\right)+P\left(x_{1}, \cdots, x_{m}\right) .
\end{aligned}
$$

Proof of (ii). As $\left[\begin{array}{l}n \\ k\end{array}\right]$ is a polynomial in $q$ with integer coefficients, and

$$
\begin{aligned}
R_{n}\left(x_{1}, \cdots, x_{m}\right) & =\prod_{j=1}^{n}\left(\left(1-q^{-j} x_{1}\right) \cdots\left(1-q^{-j} x_{m}\right)\right) \\
& =q^{-m n(n+1) / 2} \prod_{j=1}^{n}\left(\left(q^{j}-x_{1}\right) \cdots\left(q^{j}-x_{m}\right)\right),
\end{aligned}
$$

we have (2.13).

Proof of (iii). From (2.3) and (2.8), for $1 \leq k \leq n$,

$$
R_{n}\left(x_{1}, \cdots, x_{m}\right) S_{k}\left(x_{1}, \cdots, x_{m}\right)=\sum_{h=1}^{k} q^{k-h} \prod_{\substack{j=1 \\ j \neq h}}^{n}\left(\left(1-q^{-j} x_{1}\right) \cdots\left(1-q^{-j} x_{m}\right)\right),
$$

so from $(2.16)$,

$$
q^{m n(n+1) / 2} R_{n}\left(x_{1}, \cdots, x_{m}\right) S_{k}\left(x_{1}, \cdots, x_{m}\right) \in \mathbb{Z}\left[q, x_{1}, \cdots, x_{m}\right] .
$$

Now for $t<q^{-\ell}$, where $\ell>0$ is an integer such that $\left|q^{-\ell} x_{i}\right|<q$, for all $i=1, \cdots, m$,

$$
L\left(t x_{1}, \cdots, t x_{m}\right)=L^{*}\left(t x_{1}, \cdots, t x_{m}\right)=\sum_{j_{1}, \cdots, j_{m}=0}^{\infty} \frac{x_{1}^{j_{1}} \cdots x_{m}^{j_{m}} t^{j_{1}+\cdots+j_{m}}}{q^{j_{1}+\cdots+j_{m}+1}-1},
$$


then from (1.7) and (2.18), for $t<\min \left\{q^{-n}, q^{-\ell}\right\}$,

$$
\frac{L\left(t x_{1}, \cdots, t x_{m}\right)}{\prod_{k=0}^{n}\left(t-q^{-k}\right)}=(-1)^{n+1} q^{n(n+1) / 2} \sum_{j_{1}, \cdots, j_{m}, l=0}^{\infty}\left[\begin{array}{c}
n+l \\
l
\end{array}\right] \frac{x_{1}^{j_{1}} \cdots x_{m}^{j_{m}} t^{j_{1}+\cdots+j_{m}+l}}{q^{j_{1}+\cdots+j_{m}+1}-1} .
$$

So

$$
\begin{aligned}
& \frac{1}{n !} \frac{d^{n}}{d t^{n}}\left\{\frac{L\left(t x_{1}, \cdots, t x_{m}\right)}{\prod_{k=0}^{n}\left(t-q^{-k}\right)}\right\}_{t=0} \\
= & (-1)^{n+1} q^{n(n+1) / 2} \sum_{\substack{j_{1}+\cdots+j_{m}+l=n \\
0 \leq j_{1}, \cdots, j_{m}, l \leq n}}\left[\begin{array}{c}
n+l \\
l
\end{array}\right] \frac{x_{1}^{j_{1}} \cdots x_{m}^{j_{m}}}{q^{j_{1}+\cdots+j_{m}+1}-1},
\end{aligned}
$$

and (2.14) follows from (2.11), (2.17) and (2.19).

Proof of (iv). For $R>1$ and $\Gamma:=\{z:|z|=R\}$, we have from (2.9),

$$
\begin{aligned}
\left|I\left(x_{1}, \cdots, x_{m}\right)\right| & \leq R \cdot \frac{\left|R_{n}\left(x_{1}, \cdots, x_{m}\right)\right| \max _{|t|=R}\left|L\left(t x_{1}, \cdots, t x_{m}\right)\right|}{R^{n+1} \prod_{k=0}^{n}\left(R-|q|^{-k}\right)} \\
& \leq \frac{f_{q} \max _{|t|=R}\left|L\left(t x_{1}, \cdots, t x_{m}\right)\right|}{R^{n} \prod_{k=0}^{n}\left(R-q^{-k}\right)} .
\end{aligned}
$$

Now for $0<\left|x_{1}\right|, \cdots,\left|x_{m}\right|<q$,

$$
\begin{aligned}
\left|R_{n}\left(x_{1}, \cdots, x_{m}\right)\right| & =\prod_{j=1}^{n}\left|\left(1-q^{-j} x_{1}\right) \cdots\left(1-q^{-j} x_{m}\right)\right| \\
& \leq \prod_{j=0}^{\infty}\left(1+q^{-j}\right)^{m}:=f_{q}
\end{aligned}
$$

where $f_{q}$ is a constant depending only on $q$ and $m$.

Let $R=q^{m n}$. As

$$
\max _{|t|=R}\left|1-q^{-j} t x_{i}\right| \geq \max _{|t|=R}\left|1-q^{-j}\right| t|| x_{i}|| \geq\left|1-q^{m n-j+1}\right|,
$$

for $1 \leq i \leq m, j=1,2, \cdots$, and

$$
q^{j}-1=q^{j}\left(1-q^{-j}\right) \geq \frac{1}{2} q^{j},
$$

as $q$ is an integer greater than 1 , then

$$
\begin{aligned}
& \max _{|t|=R}\left|L\left(t x_{1}, \cdots, t x_{m}\right)\right| \\
\leq & \max _{|t|=R} \sum_{j=1}^{\infty}\left|\frac{q^{-j}}{\left(1-q^{-j} x_{1} t\right) \cdots\left(1-q^{-j} x_{m} t\right)}\right| \\
\leq & \left(\sum_{j=1}^{m n} \frac{q^{j-m n-1}}{\left(q^{j}-1\right)^{m}}\right)+\frac{q^{-m n-1}}{\left(1-x_{1} / q\right) \cdots\left(1-x_{m} / q\right)}+\left(\sum_{j=1}^{\infty} \frac{q^{-j-m n-1}}{\left(1-q^{-j}\right)^{m}}\right)
\end{aligned}
$$




$$
\begin{aligned}
& \leq q^{-m n-1}\left(\sum_{j=1}^{m n-1} \frac{2^{m}}{q^{(m-1) j}}+\frac{1}{\left(1-x_{1} / q\right) \cdots\left(1-x_{m} / q\right)}+L(1, \cdots, 1)\right) \\
& \leq q^{-m n-1}\left(2^{m}+\frac{1}{\left(1-x_{1} / q\right) \cdots\left(1-x_{m} / q\right)}+L(1, \cdots, 1)\right) \\
& =: C_{1} q^{-m n}
\end{aligned}
$$

where $C_{1}:=2^{m} q+\frac{q}{\left(1-x_{1} / q\right) \cdots\left(1-x_{m} / q\right)}+q L(1, \cdots, 1)$ is a constant depending only on $q, m$, and $x_{1}, \cdots, x_{m}$. Now

$$
\begin{aligned}
R^{n} \prod_{k=0}^{n}\left(R-q^{-k}\right) & =R^{2 n+1} \prod_{k=0}^{n}\left(1-q^{-n-k}\right) \\
& \geq R^{2 n+1} \prod_{j=0}^{\infty}\left(1-q^{-j}\right) \\
& \geq C_{2} q^{m n(2 n+1)}
\end{aligned}
$$

where $C_{2}:=\prod_{j=0}^{\infty}\left(1-q^{-j}\right)$ is a constant depending only on $q$. Putting (2.22) and (2.23) into (2.20), we have

$$
\left|I\left(x_{1}, \cdots, x_{m}\right)\right| \leq c_{q} q^{-2 m n(n+1)},
$$

where

$$
c_{q}:=f_{q} C_{1} / C_{1} .
$$

This completes the proof of Theorem 2.1.

\section{Proof of Theorem 1.1}

We first prove that for $0<x_{1}, \cdots, x_{m}<q$, and $q>1$,

$$
\left|I\left(x_{1}, \cdots, x_{m}\right)\right| \neq 0
$$

where $I\left(x_{1}, \cdots, x_{m}\right)$ is defined by (2.9). Note that if we choose the contour in (2.9) to be $\Gamma=\{z \in \mathbb{C}:|z|=1+\epsilon\}$, where $\epsilon>0$ is small enough such that $0<\left|t x_{1}\right|, \cdots,\left|t x_{m}\right|<q$, for $t \in \Gamma$, then

$$
L\left(t x_{1}, \cdots, t x_{m}\right)=L^{*}\left(t x_{1}, \cdots, t x_{m}\right), \quad t \in \Gamma \text {. }
$$

Now

$$
R_{n}\left(x_{1}, \cdots, x_{m}\right)=\prod_{j=1}^{n}\left(\left(1-q^{-j} x_{1}\right) \cdots\left(1-q^{-j} x_{m}\right)\right)>0
$$


for $0<x_{1}, \cdots, x_{m}<q$, and

$$
\begin{aligned}
I\left(x_{1}, \cdots, x_{m}\right)=\frac{R_{n}\left(x_{1}, \cdots, x_{m}\right)}{2 \pi i} \int_{\Gamma} \frac{L\left(t x_{1}, \cdots, t x_{m}\right) d t}{t^{2 n+2}\left(\prod_{k=0}^{n}\left(1-1 /\left(q^{k} t\right)\right)\right.} \\
=\frac{R_{n}\left(x_{1}, \cdots, x_{m}\right)}{2 \pi i} \int_{\Gamma} \frac{L\left(t x_{1}, \cdots, t x_{m}\right)}{t^{2 n+2}}\left(\sum_{j_{0}, \cdots, j_{n} \geq 0} \prod_{k=0}^{n}\left(\frac{1}{q^{k} t}\right)^{j_{k}}\right) d t \\
=R_{n}\left(x_{1}, \cdots, x_{m}\right) \sum_{j_{0}, \cdots, j_{n} \geq 0} q^{-\sum_{k=0}^{n} k j_{k}} \cdot \frac{1}{2 \pi i} \int_{\Gamma}\left\{\frac{1}{t^{2 n+2+\left(j_{0}+\cdots j_{n}\right)}}\right. \\
\left.\quad \sum_{i_{1}, \cdots i_{m}=0}^{\infty} \frac{x_{1}^{i_{1}} \cdots x_{m}^{i_{m}} t^{i_{1}+\cdots+i_{m}}}{q^{i_{1}+\cdots+i_{m}+1}-1}\right\} d t \\
=R_{n}\left(x_{1}, \cdots, x_{m}\right) \sum_{j_{0}, \cdots, j_{n} \geq 0} q^{-\sum_{k=0}^{n} k j_{k}} \\
\quad \sum_{i_{1}+\cdots+i_{m}-\left(2 n+j_{0}+\cdots j_{n}+2\right)=-1} \frac{x_{1}^{i_{1}} \cdots x_{m}^{i_{m}}}{q^{i_{1}+\cdots+i_{m}+1}-1} \\
=R_{n}\left(x_{1}, \cdots, x_{m}\right) \sum_{i_{1}+\cdots+i_{m}=2 n+j_{0}+\cdots j_{n}+1}^{q q u a d ; j_{0}, \cdots, j_{n} \geq 0} \\
>0, \quad q^{-\sum_{k=0}^{n} k j_{k}} \frac{x_{1}^{i_{1}} \cdots x_{m}^{i_{m}}}{q^{i_{1}+\cdots+i_{m}+1}-1}
\end{aligned}
$$

as $x_{1}, \cdots, x_{m} \geq 0, q>1$, and as infinitely many terms above are positive, so (3.1) holds.

Now let $r_{1}, r_{2}, \cdots, r_{m}$ be any fixed positive rational numbers such that $r_{1}, r_{2}, \cdots$, $r_{m} \neq q^{j}$ for all $j=1,2, \cdots$. From (2.7), we can see that the irrationality of $L\left(r_{1}, r_{2}, \cdots, r_{m}\right)$ is equivalent to the irrationality of $L\left(q^{-k} r_{1}, q^{-k} r_{2}, \cdots, q^{-k} r_{m}\right)$ for any integer $k \geq 1$, so we can assume that $0<r_{1}, r_{2}, \cdots, r_{m}<q$, and then

$$
L\left(r_{1}, r_{2}, \cdots, r_{m}\right)=\sum_{j_{1}, \cdots, j_{m}=0}^{\infty} \frac{r_{1}^{j_{1}} \cdots r_{m}^{j_{m}}}{q^{j_{1}+\cdots+j_{m}+1}-1}>0 .
$$

Now let

$$
H_{m, n}(q):=q^{(m-1) n(n+1) / 2}\left(\prod_{j=1}^{n+1}\left(q^{j}-1\right)\right) .
$$

Then

$$
0<\left|H_{m, n}(q)\right| \leq q^{(m n+2)(n+1) / 2}
$$

and

$$
H_{m, n}(q) \cdot\left\{Q\left(r_{1}, \cdots, r_{m}\right), P\left(r_{1}, \cdots, r_{m}\right)\right\} \subset \mathbb{Z}\left[q, r_{1}, \cdots, r_{m}\right]
$$

Now as

$$
\begin{aligned}
\Delta_{m, n} & :=\left|H_{m, n}(q) Q\left(r_{1}, \cdots, r_{m}\right) L\left(r_{1}, \cdots, r_{m}\right)+H_{m, n}(q) P\left(r_{1}, \cdots, r_{m}\right)\right| \\
& =\left|H_{m, n}(q)\right|\left|I\left(r_{1}, \cdots, r_{m}\right)\right| \\
& >0
\end{aligned}
$$


and from (2.15) and (3.3), we have

$$
\begin{aligned}
\Delta_{m, n} & \leq q^{(m n+2)(n+1) / 2} \frac{c_{q}}{q^{2 m n(n+1)}} \\
& =\frac{c_{q}}{q^{3 m n(n+1) / 2-1}} \\
& \leq \frac{c_{q}}{q^{m n^{2}}} .
\end{aligned}
$$

Finally, if

$$
r_{1}:=\frac{i_{1}}{l_{1}}, r_{2}:=\frac{i_{2}}{l_{2}}, \cdots, r_{m}:=\frac{i_{m}}{l_{m}},
$$

with $i_{1}, \cdots, i_{m}$ and $l_{1}, \cdots, l_{m}$ positive integers, then

$$
Q^{*}\left(r_{1}, \cdots, r_{m}\right):=\left(l_{1} \cdots l_{m}\right)^{2 n} H_{m, n}(q) Q\left(r_{1}, \cdots, r_{m}\right)
$$

and

$$
P^{*}\left(r_{1}, \cdots, r_{m}\right):=\left(l_{1} \cdots l_{m}\right)^{2 n} H_{m, n}(q) P\left(r_{1}, \cdots, r_{m}\right)
$$

are integers, and by (3.6) to (3.10),

$$
\begin{aligned}
0 & <\left|Q^{*}\left(r_{1}, \cdots, r_{m}\right) L\left(r_{1}, \cdots, r_{m}\right)+P^{*}\left(r_{1}, \cdots, r_{m}\right)\right| \\
& =\left(l_{1} \cdots l_{m}\right)^{2 n}\left|H_{m, n}(q)\right|\left|Q\left(r_{1}, \cdots, r_{m}\right) L\left(r_{1}, \cdots, r_{m}\right)+P\left(r_{1}, \cdots, r_{m}\right)\right| \\
& \leq\left(l_{1} \cdots l_{m}\right)^{2 n} \frac{c_{q}}{q^{m n^{2}}},
\end{aligned}
$$

which tends to zero as $n \rightarrow \infty$. This shows that $L\left(r_{1}, \cdots, r_{m}\right)$ is irrational, that is,

$$
\sum_{j_{1}, \cdots, j_{m}=0}^{\infty} \frac{r_{1}^{j_{1}} \cdots r_{m}^{j_{m}}}{q^{j_{1}+\cdots+j_{m}+1}-1}
$$

is irrational for $q>1$ integer, $r_{1}, r_{2}, \cdots, r_{m}$ positive rationals less than $q$ and integer $m \geq 1$, and

$$
\sum_{j=1}^{\infty} \frac{q^{-j}}{\left(1-q^{-j} r_{1}\right)\left(1-q^{-j} r_{2}\right) \cdots\left(1-q^{-j} r_{m}\right)}
$$

is irrational for $q>1$ integer, $r_{1}, r_{2}, \cdots, r_{m}$ positive rationals such that $r_{1}, r_{2}, \cdots$, $r_{m} \neq q^{j}$ for all $j=1,2, \cdots$, and integer $m \geq 1$.

This completes the proof of Theorem 1.1.

Now by the standard methods (as in chapter 11 of Borwein and Borwein [1), the estimates in the proof of Theorem 1.1 gives that, under the assumption of the theorem,

$$
\left|L\left(r_{1}, \cdots, r_{m}\right)-\frac{s}{t}\right|>\frac{1}{t^{\alpha}},
$$

for some constant $\alpha$ and all integers $s$ and $t$, and hence

$$
\sum_{j_{1}, \cdots, j_{m}=0}^{\infty} \frac{r_{1}^{j_{1}} \cdots r_{m}^{j_{m}}}{q^{j_{1}+\cdots+j_{m}+1}-1}
$$

is not a Liouville number. 


\section{REFERENCES}

[1] J.M.Borwein and P.B.Borwein, Pi and the AGM-A Study in Analytic Number Theory and Computational Complexity, New York, 1987.

[2] P.B.Borwein, Padé Approximants for the q-Elementary Functions, Constr. Approx. 4(1988), 391-402. MR 89f:41022

[3] P.B.Borwein, On the Irrationality of $\sum\left(1 /\left(q^{n}+r\right)\right)$, J. of Number Theory 37(1991), 253-259. MR 92b: 11046

[4] P.B.Borwein and P.Zhou, On the Irrationality of A Certain q Series, Proc. AMS 127(1999), 1605-1613. MR 99i:11057

[5] D.V.Chudnovsky and G.V.Chudnovsky, Padé and Rational Approximation to Systems of Functions and Their Arithmetic Applications, In "Lecture Notes in Mathematics," Vol. 1052, Springer-Verlag, Berlin, 1984. MR 86a:11029

[6] P.Erdös, On Arithmetical Properties Of Lambert Series, J. Indian Math. Soc. (N.S.) 12(1948), 63-66. MR 10:594c

[7] P.Erdös and R.L.Graham, Old and New Problems and Results in Combinatorial Number Theory, Enseign. Math. Monograph, 28(1980). MR 82j:10001

[8] G.Gasper and M.Rahman, Basic Hypergeometric Series, Encyclopaedia of Maths and its Applications, Vol. 35, Cambridge University Press, Cambridge, 1990. MR 91d:33034

[9] K.Mahler, Zur Approximation der Exponentialfunktion und des Logarithmus, J. Reine Angew. Math. 166(1931), 118-150.

[10] R.Wallisser, Rationale Approximation des q-Analogons der Exponential-funktion und Irrationalitätsaussagen für diese Funktion, Arch. Math. 44 (1985), 59-64. MR 86i:11036

[11] P.Zhou and D.S.Lubinsky, On the Irrationality of the Infinite Product $\prod_{j=0}^{\infty}\left(1 \pm q^{-j} r+q^{-2 j} s\right)$, Analysis 17 (1997), 129-153. MR 99c:11089]

Department of Mathematics and Statistics, Simon Fraser University, Burnaby, British Columbia, Canada V5A 1S6

E-mail address: pborwein@cecm.sfu.ca

Department of Mathematics, Statistics \& Computer Science, St. Francis Xavier University, Antigonish, Nova Scotia, Canada B2G 2W5

E-mail address: pzhou@stfx.ca 